\title{
Importance of excision repair cross-complementation group 1 and ribonucleotide reductase $M 1$ as prognostic biomarkers in malignant pleural mesothelioma treated with platinum-based induction chemotherapy followed by surgery
}

Lukas Frischknecht, MD, ${ }^{\mathrm{a}}$ Mayura Meerang, PhD, ${ }^{\mathrm{b}}$ Alex Soltermann, MD, ${ }^{\mathrm{a}}$ Rolf Stahel, MD, Holger Moch, MD, ${ }^{a}$ Burkhardt Seifert, $\mathrm{PhD},{ }^{\mathrm{d}}$ Walter Weder, $\mathrm{MD},{ }^{\mathrm{b}}$ and Isabelle Opitz, $\mathrm{MD}^{\mathrm{b}}$

Objectives: Survival and response to platinum-based induction chemotherapy are heterogeneous among patients with malignant pleural mesothelioma. The aim of the present study was to assess the prognostic role of DNA repair markers, such as excision repair cross-complementation group 1 and ribonucleotide reductase M1, in multimodally treated patients with malignant pleural mesothelioma.

Methods: Tumor tissue of a malignant pleural mesothelioma cohort $(\mathrm{n}=107)$ treated with platinum/ gemcitabine $(\mathrm{n}=46)$ or platinum/pemetrexed $(\mathrm{n}=61)$ induction chemotherapy followed by extrapleural pneumonectomy was assembled on a tissue microarray. Immunohistochemical expression of excision repair cross-complementation group 1 (nuclear) and ribonucleotide reductase M1 (nuclear and cytoplasmic) was assessed for its prognostic impact (association with overall survival or freedom from recurrence).

Results: Patients with high nuclear ribonucleotide reductase M1 expression before chemotherapy showed significantly longer freedom from recurrence $(P=.03)$. When specifically analyzed in the subgroup of patients receiving platinum/gemcitabine followed by extrapleural pneumonectomy, high nuclear ribonucleotide reductase M1 was associated with prolonged freedom from recurrence $(P=.03)$ and overall survival $(P=.02)$. Low excision repair cross-complementation group 1 expression in prechemotherapy tumor tissues was associated with significantly longer freedom from recurrence $(P=.04)$. Nuclear ribonucleotide reductase M1 and excision repair cross-complementation group 1 were independent prognosticators of freedom from recurrence in addition to $\mathrm{pT}$ stage in multivariate analysis.

Conclusions: In the present study, nuclear ribonucleotide reductase M1 and excision repair crosscomplementation group 1 expression were identified as independent prognosticators for freedom from recurrence of malignant pleural mesothelioma in patients undergoing induction chemotherapy followed by extrapleural pneumonectomy. (J Thorac Cardiovasc Surg 2015;149:1539-47)

See related commentary on pages 1548-9.

Supplemental material is available online.

\footnotetext{
From the Institute of Surgical Pathology, ${ }^{\mathrm{a}}$ Division of Thoracic Surgery, ${ }^{\mathrm{b}}$ and Laboratory of Molecular Oncology, ${ }^{c}$ University Hospital Zurich, Zurich, Switzerland; and Division of Biostatistics, ${ }^{\text {d }}$ ISPM, University Zurich, Zurich, Switzerland. Funded by the Krebsliga Zurich grant, SNSF Professorship to Dr Opitz.

Read at the 94th Annual Meeting of The American Association for Thoracic Surgery, Toronto, Ontario, Canada, April 26-30, 2014.

Received for publication April 9, 2014; revisions received Dec 11, 2014; accepted for publication Jan 23, 2015; available ahead of print April 1, 2015.

Address for reprints: Isabelle Opitz, MD, Division of Thoracic Surgery, University Hospital Zürich, Rämistrasse 100, CH 8091 Zürich, Switzerland (E-mail: isabelle.schmitt-opitz@usz.ch).

$0022-5223 / \$ 36.00$

Copyright (c) 2015 by The American Association for Thoracic Surgery http://dx.doi.org/10.1016/j.jtcvs.2015.01.065
}

The best outcomes for patients with malignant pleural mesothelioma (MPM) are reported after multimodal approaches, including macroscopic complete resection by extrapleural pneumonectomy (EPP) or extended pleurectomy/decortication. $^{1,2}$ One particular multimodal approach is induction chemotherapy with platinum (cisplatin or carboplatin) combined with gemcitabine or pemetrexed followed by EPP and radiotherapy. ${ }^{3-5}$ However, survival after this treatment varies significantly with some patients surviving months or even years beyond the current median survival, whereas others do not seem to benefit. This highlights that additional factors prognosticating survival outcomes accompanying the decision are required.

Excision repair cross complementation group 1 (ERCC1) plays an important role in several DNA repair pathways, especially nucleotide excision repair of ultraviolet-induced thymine dimers or platinum adducts. ${ }^{6}$ ERCC1 has been extensively studied as a prognostic and predictive marker in non-small cell lung cancer (NSCLC). ${ }^{7,8}$ 


$$
\begin{aligned}
& \text { Abbreviations and Acronyms } \\
& \begin{aligned}
\text { EPP }= & \text { extrapleural pneumonectomy } \\
\text { ERCC1 } & \text { excision repair cross-complementation } \\
& \text { group } 1 \\
\text { FFR }= & \text { freedom from recurrence } \\
\text { MPM } & \text { malignant pleural mesothelioma } \\
\text { NSCLC }= & \text { non-small cell lung cancer } \\
\text { OS } & =\text { overall survival } \\
\text { RRM1 } & =\text { ribonucleotide reductase M1 } \\
\text { TMA }= & \text { tissue microarray }
\end{aligned}
\end{aligned}
$$

Ribonucleotide reductase M1 (RRM1) is the catalytic subunit of ribonucleotide reductase, the enzyme responsible for the de novo synthesis of most of the deoxyribonucleotides. Elevated levels of RRM1 were shown to increase the DNA repair capacity. ${ }^{9}$ Similar to the observations with ERCC1, patients with high RRM1 levels had a better prognosis if they received no chemotherapy. ${ }^{6}$ Because the chemotherapeutic agent gemcitabine is known to interfere with human ribonucleotide reductase activity, the prognostic value of RRM1 has been studied mainly in patients with NSCLC treated with gemcitabine-based regimens.

Platinum-based compounds, also in combination with gemcitabine, represent the cornerstone of chemotherapy for patients with MPM. To date, there are conflicting data in the literature about the prognostic role of ERCC1 expression in patients with MPM. ${ }^{10-12}$ In a small cohort of 80 patients with MPM, Kao and colleagues ${ }^{10}$ showed a trend toward better survival for high ERCC1 expression. To the contrary, negative ERCC1 expression was associated with long progression-free survival in a different study in patients treated with cisplatin/vinorelbine. ${ }^{11}$ Another study with patients treated with carboplatin/pemetrexed did not find any predictive significance for ERCC $1 .{ }^{12}$ To date, there is no study investigating immunohistochemical staining of RRM1 and its association with clinical outcome and therapy response in patients with MPM.

The purpose of the present study was to investigate whether ERCC1 and RRM1 expression is a prognostic factor for clinical outcomes of patients uniformly treated with induction chemotherapy followed by EPP.

\section{MATERIALS AND METHODS \\ Patients}

An MPM cohort was set up consisting of 107 patients (1999-2009) uniformly treated with induction chemotherapy followed by EPP as described previously ${ }^{3,13}$ (Figure E1). Patients were followed in our outpatient clinic with regular clinical visits, including a computed tomography scan. Of the present cohort, 3 patients $(2 \%)$ were lost to follow-up. The study was approved and waivers of consent were granted by the Ethical Committee Zurich (StV 29-2009 and EK-ZH 2012-0094).

\section{Tissue Microarray Construction and Immunohistochemistry}

A set of 4 (2 for pretreatment and 2 for post-treatment samples) tissue microarrays (TMAs) were fabricated with a custom-made, semiautomatic tissue arrayer (Beecher Instruments, Sun Prairie, Wis) as described previously. ${ }^{14}$ Four punches (0.6 mm in diameter) were taken from postchemotherapy EPP specimen samples. Two punches from prechemotherapy diagnostic samples were collected on the TMA. Deparaffinized 2- $\mu$ m-thick TMA sections were automatically stained with BenchMark (Ventana Medical Systems, Tucson, Ariz) using the iView DAB Detection kit (Ventana Medical Systems). The primary antibodies were a mouse monoclonal antibody specific against the fulllength human ERCC1 protein at a 1:100 dilution (clone 8F1; Abcam, Cambridge, Mass) and a rabbit anti RRM1 polyclonal antibody (Protein Tech Group cat. 10526-1-AP, Chicago, Ill) diluted 1:100.

Immunohistochemical evaluation of the TMAs was conducted by 2 observers (S.T. and L.F.). The staining intensity (for ERCC1 nuclear and for RRM1 cytoplasmic and nuclear) was semiquantitatively scored 0 (negative), 1 (weak), 2 (moderate), or 3 (strong). Further, the percentage of cells having any positivity was proportionally scored $0(0 \%), 0.1(1 \%-10 \%)$, $0.5(11 \%-50 \%)$, or $1.0(\geq 51 \%)$. An $\mathrm{H}$-score was calculated by multiplying the intensity with the frequency as described previously. ${ }^{8}$ TMA spots that lacked tumor tissue because of sampling error, damaged tissue, or lack of tissue at some array positions were excluded from the analysis, leading to different patient numbers in the subsequent analysis.

\section{Statistical Analysis}

ERCC1 and RRM1 expression were assessed and associated with histologic subtype, modified Response Evaluation Criteria In Solid Tumors criteria, pT or pN stage, and International Mesothelioma Interest Group stages using the Kruskal-Wallis test and Mann-Whitney $U$ test. To evaluate the change of expression of ERCC1 and RRM1 during chemotherapy (in paired pre- and postchemotherapy samples), the Wilcoxon signed-rank test was used. Tumor response to chemotherapy treatment was evaluated on the basis of modified Response Evaluation Criteria In Solid Tumors criteria. ${ }^{15}$ For survival analysis, the calculated $\mathrm{H}$-scores were dichotomized closest to the median in the tumor samples undergoing similar treatment (ie, pre- and postchemotherapy). Freedom from recurrence (FFR) was defined as the time interval between the start of the first cycle of chemotherapy and the recurrence of the tumor based on the first confirmed sign of disease (clinically, histologically confirmed by biopsy or fine-needle aspiration, or radiologically evident in serial follow-up imaging with increasing tumor masses). Overall survival (OS) represents survival from the first cycle chemotherapy to last follow-up or time-point of death. ${ }^{16} \mathrm{Me}$ dian survival time was assessed by Kaplan-Meier curves, and the influence of ERCC1 and RRM1 expression was analyzed by log-rank test. To test the independence of different factors on survival, a stepwise Cox regression was performed including all prognostic factors being significant in the univariate analysis: gender, age ( $\leq 61$ vs $>61$ years), pT (categoric variable: pT1 vs pT2, pT1 vs pT3, pT1 vs pT4), pN (pN0 vs pN1/2), nuclear RRM1 (prechemotherapy), and nuclear ERCC1 (prechemotherapy). Statistical analyses were carried out using IBM SPSS Statistics for Windows, version 22.0 (IBM Corp, Armonk, NY). Confidence limits of the Kaplan-Meier at 1, 2, and 3 years of OS and FFR, respectively, were calculated using STATA version 13.1 (StataCorp LP, College Station, Tex).

\section{RESULTS}

Expression of Ribonucleotide Reductase M1 and Excision Repair Cross-Complementation Group 1 and Association With Clinicopathologic Data

RRM1 is expressed in both the cytoplasmic and the nuclear cell compartments (Figure 1). The nuclear expression is low in general, with a median $\mathrm{H}$-score of 0 in both preand postchemotherapy tissues (Table E1). ERCC1 was 


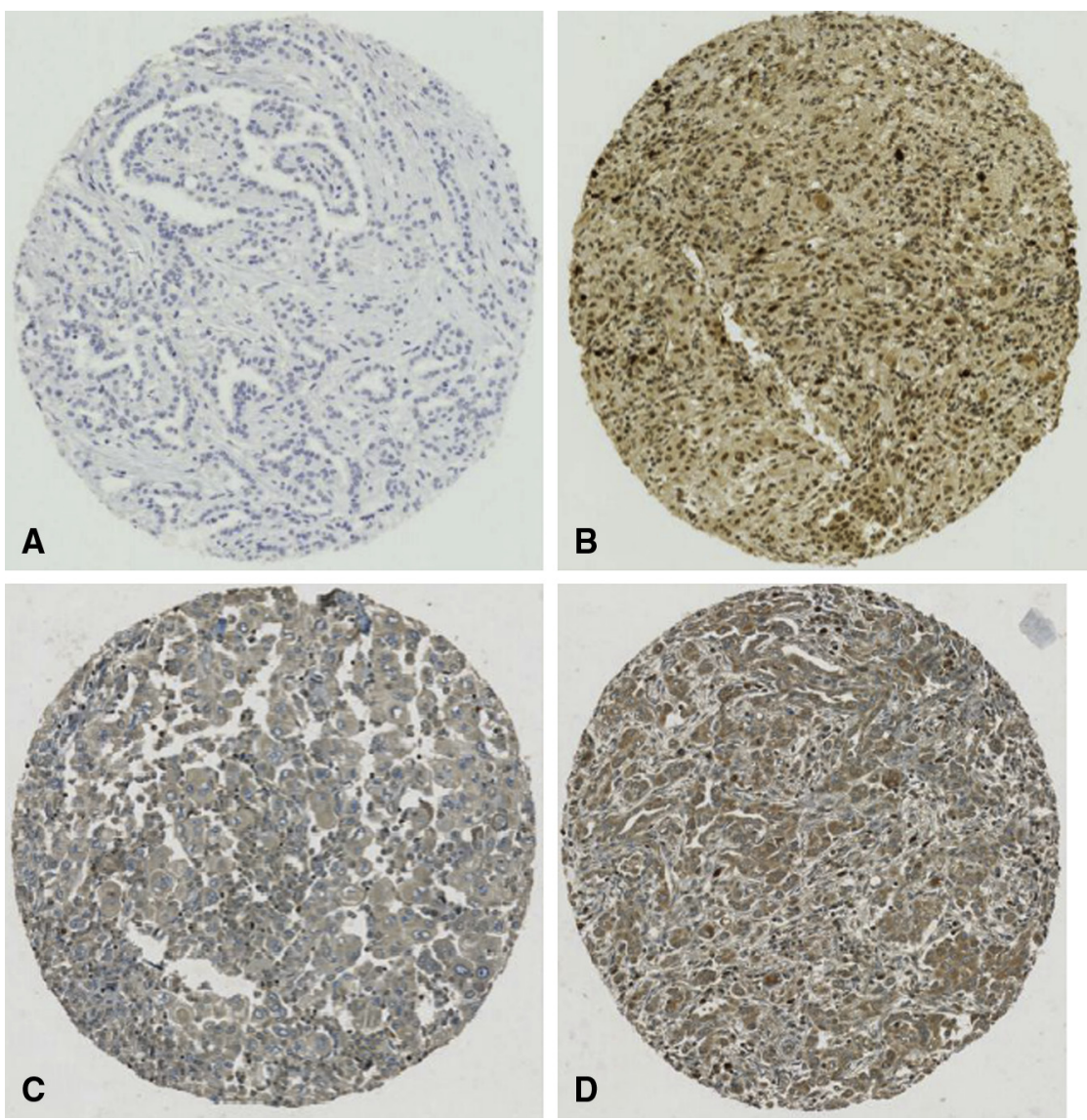

FIGURE 1. Immunohistochemical staining of ERCC1 and RRM1: examples of low (A) and high (B) ERCC1 staining, and low (C) and high (D) cytoplasmic RRM1.

mainly localized in the nucleus with a median $\mathrm{H}$-score of 2 (Table E1). The patients' characteristics and clinicopathologic data and their association with FFR and OS are summarized in Table 1. We found that gender, age, histotype, $\mathrm{T}$ stage, and $\mathrm{N}$ stage are associated with OS and FFR (Table 1). Expression of prechemotherapy nuclear RRM1 was significantly increased in patients with stage pT4 (Kruskal-Wallis test, $P=.045$ ) and International Mesothelioma Interest Group IV (Kruskal-Wallis test, $P=.02$ ) (data not shown). No association between nuclear/cytoplasmic RRM1 or nuclear ERCC1 expression and histologic subtype was found (data not shown).

\section{Induction Chemotherapy Induced Change in Expression of Markers}

Cytoplasmic RRM1 increased significantly after induction chemotherapy $(\mathrm{n}=64$ paired samples, Wilcoxon signed-rank test, $P<.0005)$, whereas nuclear RRM1 decreased significantly after chemotherapy $(\mathrm{n}=64$ paired samples, Wilcoxon signed-rank test, $P<.0005)$ (Figure 2). Nuclear ERCC1 was not significantly changed after chemotherapy $(\mathrm{n}=84$ paired samples, Wilcoxon signed-rank test, $P=.07)$.

\section{Significance of Ribonucleotide Reductase M1 as a Prognostic Marker in Patients With Malignant Pleural Mesothelioma}

High nuclear RRM1 protein levels in the prechemotherapy samples of all patients $(\mathrm{n}=71)$ were significantly associated with a longer FFR $(P=.03)$ (Figure 3, $A)$. This effect also was significant in the subgroup of patients receiving platinum/gemcitabine induction chemotherapy followed by EPP ( $\mathrm{n}=32)$, where high nuclear RRM1 was associated with both longer FFR and OS $(P=.02$ and .03 , respectively) (Figure $3, B$ and $C$ ). We did not observe any association between nuclear RRM1 expression and survival of the patient subgroup receiving platinum/pemetrexed induction chemotherapy followed by EPP. There was no association between cytoplasmic RRM1 and FFR or OS. No association between RRM1 expression and response to chemotherapy was observed in our patient cohort.

\section{Significance of Excision Repair \\ Cross-Complementation Group 1 as a Prognostic Marker in Patients With Malignant Pleural Mesothelioma}

Low nuclear ERCC1 in the pretreatment biopsies was associated with a longer FFR in patients undergoing 
TABLE 1. Kaplan-Meier survival analysis for overall survival and freedom from recurrence of possible prognostic factors

\begin{tabular}{|c|c|c|c|c|}
\hline & \multicolumn{2}{|l|}{ OS } & \multicolumn{2}{|l|}{ FFR } \\
\hline & Median OS $(95 \%$ CI $)$ & $P$ value & Median FFR $(95 \%$ CI $)$ & $P$ value \\
\hline \multirow[t]{2}{*}{ Gender } & Female (9): 53 (22-84) & $.003 \dagger$ & Female (9): 27 (9-46) & $.03 \dagger$ \\
\hline & Male (98): 21 (17-24) & & Male (98): 14 (11-17) & \\
\hline \multirow[t]{2}{*}{ Age at diagnosis } & $\leq 61$ y $(59): 20(17-24)$ & .06 & $\leq 61$ y $(59): 14(10-18)$ & $.008 \dagger$ \\
\hline & $>61$ y (48): $23(18-28)$ & & $>61$ y (48): 15 (13-17) & \\
\hline \multirow[t]{2}{*}{ Histotype pre-CTX* (19 missing) } & Epithelioid (55): 23 (17-29) & $.003 \dagger$ & Epithelioid (55): 18 (14-21) & .1 \\
\hline & Nonepithelioid (33): 13 (7-19) & & Nonepithelioid (33): 15 (10-19) & \\
\hline \multirow[t]{2}{*}{ Histotype post-CTX } & Epithelioid (58): 23 (17-29) & $.02 \dagger$ & Epithelioid (58): 16 (12-19) & .3 \\
\hline & Nonepithelioid (49): 16 (12-20) & & Nonepithelioid (49): 14 (11-18) & \\
\hline \multirow[t]{2}{*}{ CTX treatment } & Platinum/gemcitabine (46): 23 (20-26) & .8 & Platinum/gemcitabine (46): 15 (12-18) & .9 \\
\hline & Platinum/pemetrexed (61): 21 (16-27) & & Platinum/pemetrexed (61): 14 (11-18) & \\
\hline \multirow[t]{3}{*}{ Response* (32 missing) } & PR (27): 23 (20-26) & .08 & PR (27): 16 (12-20) & .09 \\
\hline & SD (28): 17 (11-23) & & SD (28): $12(9-14)$ & \\
\hline & PD (20): 15 (6-24) & & PD (20): $11(8-14)$ & \\
\hline \multirow[t]{4}{*}{ pT stage } & pT1 (10): $23(0-48)$ & .1 & pT1 (10): 45 (5-86) & $.004 \dagger$ \\
\hline & pT2 (40): 22 (20-24) & & pT2 (40): 19 (15-22) & \\
\hline & pT3 (47): 21 (16-26) & & pT3 (47): $12(9-15)$ & \\
\hline & pT4 (10): 14 (9-20) & & pT4 (10): $11(7-15)$ & \\
\hline \multirow[t]{2}{*}{ pN stage* (1 missing) } & pN0 (70): 23 (19-27) & $.01 \dagger$ & pN0 (70): 16 (14-18) & $.01 \dagger$ \\
\hline & $\mathrm{pN} 1 / 2$ (36): 19 (14-24) & & pN1/2 (36): 13 (9-16) & \\
\hline \multirow[t]{2}{*}{ Adjuvant radiotherapy* (2 missing) } & No (43): $19(13-24)$ & .5 & No (43): $13(11-15)$ & .7 \\
\hline & Yes (62): 23 (20-26) & & Yes (62): 15 (13-17) & \\
\hline \multirow[t]{2}{*}{ Second-line CTX* (11 missing) } & No (54): 15 (5-26) & .1 & & \\
\hline & Yes (42): 23 (19-27) & & & \\
\hline
\end{tabular}

The analysis was performed in 107 patients undergoing induction chemotherapy followed by EPP. Data are given as median survival in months (starting from first cycle of CTX received) with $95 \%$ CI. $O S$, Overall survival; $F F R$, freedom from recurrence; $C I$, confidence interval; $C T X$, chemotherapy; $P R$, partial response; $S D$, stable disease; $P D$, progressive disease. *Data were not available for all 107 patients. $\dagger$ Significant results according to log-rank test.

induction platinum-based chemotherapy followed by EPP $(P=.04)(\mathrm{n}=87)($ Figure $3, D)$.

\section{Multivariate Analysis}

All variables with a significant impact on FFR in univariate analysis (Table 1) were submitted to multivariate analysis using the stepwise forward model. Nuclear RRM1, nuclear ERCC1, and pT stage were independent prognosticators for FFR (Table 2).

\section{DISCUSSION}

We have discovered prognostic significance of high nuclear RRM1 protein level for longer FFR in patients with MPM receiving platinum/gemcitabine and platinum/ pemetrexed induction chemotherapy followed by EPP. The prognostic role of nuclear RRM1 is more pronounced in the subgroup of patients receiving platinum/gemcitabine induction chemotherapy, in whom high nuclear RRM1 expression was significantly associated with both longer FFR and OS. In addition, we further confirmed low ERCC1 expression as a prognostic marker for longer FFR.

RRM1, a subunit of ribonucleotide reductase, plays a key role in providing precursors (deoxyribonucleotides) for the DNA synthesis and repair. We observed a longer survival of patients with MPM exhibiting high nuclear RRM1 levels in their tissues before receiving doublet induction chemotherapy. Being a major molecular target of gemcitabine, high nuclear RRM1 levels were strongly associated with better survival in the subgroup receiving the platinum/gemcitabine induction chemotherapy followed by EPP in our study cohort. In contrast to our finding, several studies in NSCLC have discovered that a low RRM1 (mRNA or protein) level was associated with better prognosis in patients with NSCLC receiving cisplatin/gemcitabine. ${ }^{17-19}$ Nevertheless, various studies in NSCLC and other solid tumors indicated discrepant results depending on the type of analysis, treatment, and patient cohort. ${ }^{20}$ Of note, most of the studies in NSCLC investigating RRM1 expression did not distinguish between nuclear and cytoplasmic localization, and many studies have relied on mRNA expression. ${ }^{21}$ In our study, most of the RRM1 protein was localized in the cytoplasmic fraction, but we could not identify any prognostic value of cytoplasmic RRM1. The protein expression of these markers also may be examined quantitatively as described. ${ }^{22}$ Nevertheless, the manual $\mathrm{H}$-scoring method allowed us to distinguish the localization of the protein (nuclear vs cytoplasmic). By using this scoring method, we could see that only the nuclear fraction of RRM1 shows a prognostic significance.

It is still an ongoing debate whether deoxynucleotide synthesis for DNA replication and repair takes place in cytoplasmic or nuclear fractions. ${ }^{23}$ Recent evidence 


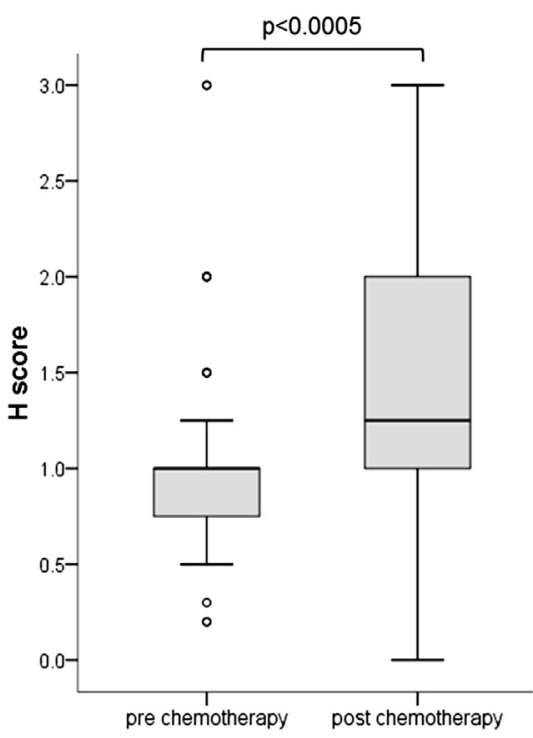

A

cytoplasmic RRM1

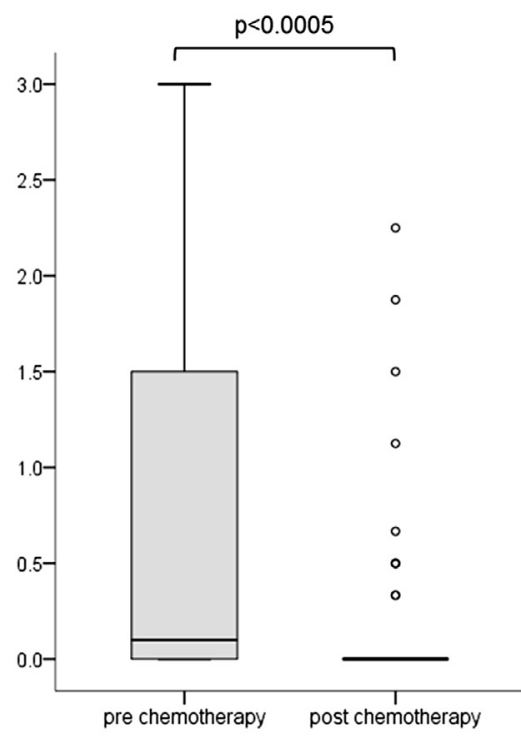

nuclear RRM1

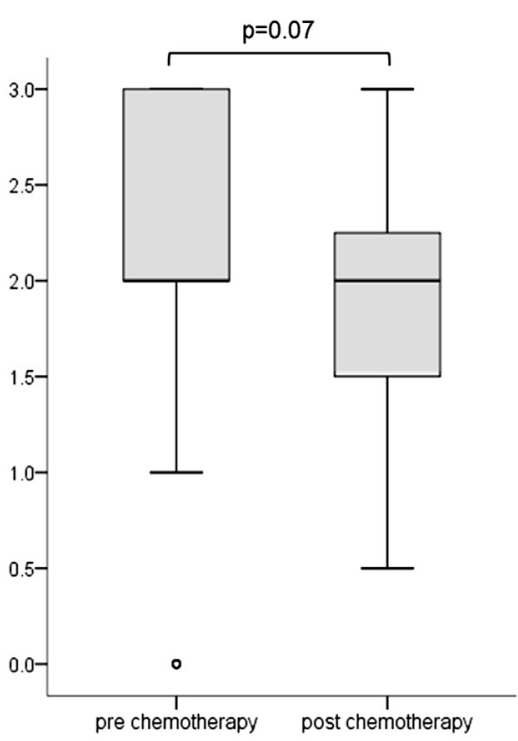

C nuclear ERCC1 Increase in cytoplasmic RRM1 expression after chemotherapy $(n=64)$ : median expression prechemotherapy: 1.00 (interquartile range [IQR], 0.75-1.00), median expression postchemotherapy: 1.25 (IQR, 1.00-2.00) $(P<.0005)$. B, Decrease in nuclear RRM1 after chemotherapy $(\mathrm{n}=64)$ : median expression prechemotherapy: 0.10 (IQR, 0.00-1.50), median expression postchemotherapy: 0.00 (IQR, 0.00-0.00) $(P<.0005)$. C, No significant decrease in nuclear ERCC1 expression after chemotherapy $(\mathrm{n}=84)$ : median expression prechemotherapy: $2.00(\mathrm{IQR}, 2.00-3.00)$, median expression postchemotherapy: 2.00 (IQR, 1.50-2.25) $(P=.07)$. RRM1, Ribonucleotide reductase M1; ERCC1, Excision repair cross-complementation group 1.

suggested that localization of ribonucleotide reductase complex (RRM1, RRM2, and p53R2) in the nucleus may play an important role in DNA repair as evidenced by translocation of active ribonucleotide reductase complex into the nucleus after DNA damage inducted by ultraviolet treatment. ${ }^{23}$ Thus, our study further indicates the biological importance of nuclear fraction of RRM1, a finding that needs further confirmation.

By comparing paired samples (pre- and postchemotherapy), we observed an increased expression of RRM1 in cytoplasm and decreased expression in nucleus after induction chemotherapy. Nevertheless, the evidence is insufficient to discuss whether the change is due to altered protein localization or to decreased total gene expression level. Also in NSCLC studies using the same RRM1 antibody as applied in the present study, changes in RRM1 protein expression after chemotherapy were discovered. $^{24,25}$ This may indicate the response of cancer cells toward a resistance phenotype, because it has been reported that RRM1 mRNA level is highly upregulated in acquired gemcitabine-resistant colon cancer cell line continuously treated with gemcitabine in vivo. ${ }^{26}$ RRM1 expression analyzed in postchemotherapy tissues did not provide prognostic significance.

We showed that low ERCC1 expression in the pretreatment tissues of patients undergoing treatment with induction chemotherapy (platinum/gemcitabine or platinum/pemetrexed) followed by EPP was significantly associated with a longer FFR. A low level of ERCC1 was previously reported to be associated with sensitivity to cisplatin for NSCLC and therefore with increased survival. ${ }^{6}$ Only a few studies on MPM were reported with inconsistent findings. ${ }^{10-12,27,28}$ In patients receiving cisplatin/ vinorelbine, a longer FFR was observed in case of ERCC1 negativity $(\mathrm{n}=30)$ compared with ERCC1 positivity $(\mathrm{n}=20) .{ }^{11}$ Similar results were shown by Ting and colleagues $^{28}$ in a cohort of 103 patients receiving first-line chemotherapy. The only study showing different results was by Zucali and colleagues, ${ }^{12}$ who could not show any effect of ERCC1 expression on clinical outcome. However, the study includes 12 post-treatment (carboplatin/pemetrexed) tissues in the analysis (a total of 99)..$^{12}$ In contrast, we cannot confirm any association between ERCC1 expression in post-therapy samples and clinical outcome in the present analysis. In the subgroup of patients receiving platinum/ gemcitabine induction chemotherapy followed by EPP, low ERCC1 was associated with a significantly better response to therapy (data not shown). Because of the limited number of patients in this subgroup, it is important to further confirm in a bigger patient cohort whether ERCC1 has a role in the prediction of response to this treatment. Taken together, our study provides supporting evidence that ERCC1 is a prognostic factor for patients with MPM treated with a platinum-based regimen.

ERCC1 was described as a prognostic factor for NSCLC cohorts of mainly untreated patients. ${ }^{6}$ Therefore, we used an untreated historical cohort of MPM to explore the prognostic significance of ERCC1 without confounding 

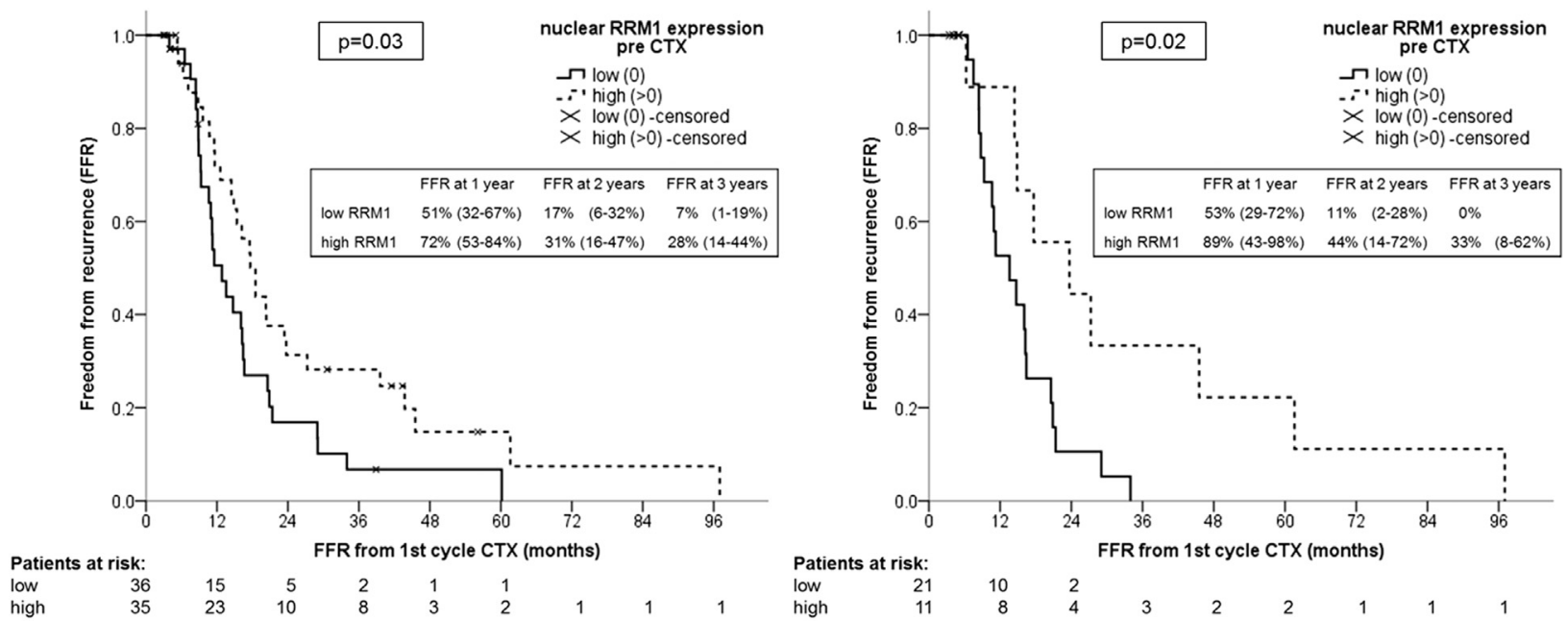

A

B
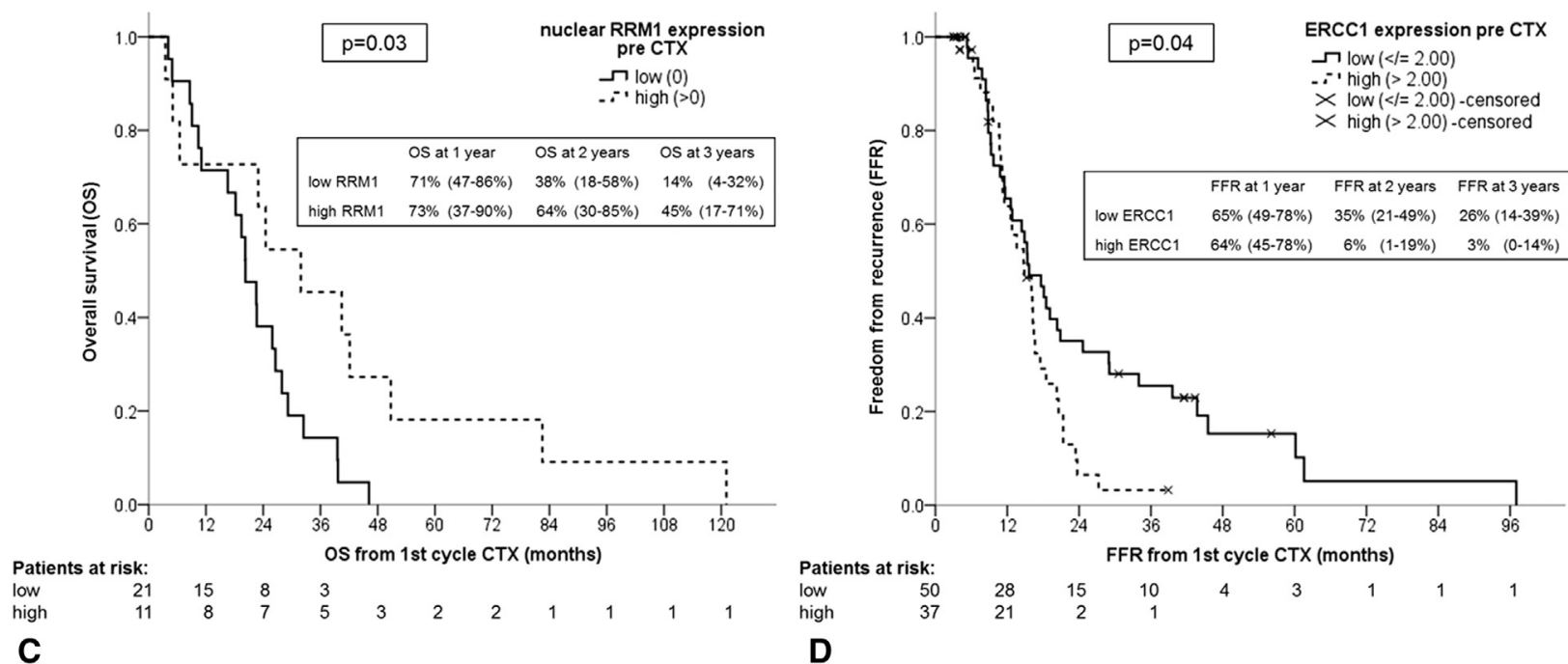

FIGURE 3. Kaplan-Meier survival curves according to dichotomized marker expression. The tables show the FFR/OS with $95 \%$ confidence interval at 1 , 2, and 3 years. A, Expression of nuclear RRM1 in prechemotherapy biopsies is associated with a prolonged FFR (no RRM1 expression [n $=36$ ]: median FFR 13 months [95\% CI, 10-16], RRM1 expression [ $\mathrm{n}=35$ ]: median FFR 19 months [95\% CI, 15-22], log-rank test $P=.03$ [ $\mathrm{n}=71]$ ). In the subgroup receiving platinum/gemcitabine treatment, both the FFR (B) and OS (C) were significantly longer in the patients expressing nuclear RRM1 in their pretreatment biopsies: no RRM1 expression ( $\mathrm{n}=21)$ : median FFR 14 months (95\% CI, 8-19), RRM1 expression $(\mathrm{n}=11)$ : median FFR 24 months (95\% CI, 6-41), $P=.02$; no RRM1 expression ( $=21)$ : median OS 20 months (95\% CI, 16-25), RRM1 expression ( $=11)$ : median OS: 32 months (95\% CI, 13-51), $P=.03$. D, Low expression of nuclear ERCC1 in prechemotherapy biopsies is associated with longer FFR (low ERCC1 [ $\leq 2.00]$ : median FFR 16 months [95\% CI, 12-20], high ERCC1 expression [>2.00]: median FFR 15 months [95\% CI, 11-19], $P=.04$ ). RRM1, Ribonucleotide reductase M1; $C T X$, chemotherapy; FFR, freedom from recurrence; $O S$, overall survival; ERCC1, excision repair cross-complementation group 1.

by chemotherapy. In line with these findings, the current study observed a strong association between high ERCC1 protein expression and longer survival when analyzed in the 43 untreated patients (data not shown).

There is recent controversy about the validity of immunohistochemical assessment of ERCC1 expression in NSCLC. The group of Jean-Charles Soria originally describing the predictive value of ERCC1 in NSCLC ${ }^{8}$ could not validate their results in a follow-up study or on the original data set $^{29}$ because of a change in the performance of the widely used ERCC1 antibody 8F1, which was also used in our study. ERCC1 exists in 4 isoforms, and only some isoforms are functional. ${ }^{29}$ The antibody we used $(8 \mathrm{~F} 1)$ for ERCC1 staining was shown to recognize all isoforms. ${ }^{29}$ RRM1 exists in only 1 isoform but has many polymorphisms that were described to associate with gemcitabine treatment efficacy and toxicity in MPM. ${ }^{30}$ This indicates the difficulty to base a therapeutic decision only on immunohistochemical assessment of marker expression. Therefore, a combination of several markers including clinical variables is needed. 
TABLE 2. Multivariate analysis of freedom from recurrence

\begin{tabular}{lcc}
\hline & HR $(\mathbf{9 5} \% \mathbf{C I})$ & $\boldsymbol{P}$ value \\
\hline $\begin{array}{l}\text { Nuclear RRM1 pre-CTX } \\
\quad \text { (expression vs no expression) }\end{array}$ & $0.4(0.2-0.8)$ & .005 \\
Nuclear ERCC1 pre-CTX (high expression & $2.2(1.2-4.0)$ & .01 \\
$\quad(>2.0)$ vs low expression $(\leq 2.0))$ & & \\
pT stage & & .001 \\
pT2 vs pT1 & $2.1(0.7-6.8)$ & .2 \\
pT3 vs pT1 & $5.3(1.7-16.7)$ & .005 \\
pT4 vs pT1 & $13.3(2.8-62.2)$ & .001 \\
\hline
\end{tabular}

The analysis was conducted using forward stepwise likelihood ratio. Parameters included in the analysis are gender, age ( $\leq 61 \mathrm{vs}>61$ years), $\mathrm{pT}$ (categoric variable: pT1 vs pT2, pT1 vs pT3, pT1 vs pT4), pN (pN0 vs pN1/2), nuclear RRM1 (pre-CTX), and nuclear ERCC1 (pre-CTX). CI, Confidence interval; RRMI, ribonucleotide reductase $\mathrm{M} 1 ; E R C C 1$, excision repair cross-complementation group 1; CTX, chemotherapy; $H R$, hazard ratio.

\section{Study Limitations}

Limitations of the present study include the small sample size of the patients receiving platinum/gemcitabine compared with platinum/pemetrexed induction chemotherapy. For TMA construction, prechemotherapy samples were composed of 2 tissue cores and postchemotherapy samples were composed of 4 cores. This number may not be sufficient to represent the total protein expression of patients with MPM with well-known intratumoral heterogeneity. Also, the different histotypes of mesothelioma have a heterogeneous behavior clinically and biologically. Therefore, we analyzed epithelioid and nonepithelioid subgroups separately, but we observed the same prognostic impact of ERCC1 and RRM1 expression in the epithelioid subtype (data not shown). No prognostic significance was observed in nonepithelioid subtypes, but this may be due to the small sample size.

\section{CONCLUSIONS}

We investigated the prognostic impact of RRM1 and ERCC1 in patients with MPM receiving doublet induction chemotherapy followed by EPP. We discovered nuclear RRM1 as a novel independent prognostic factor for patients with MPM receiving induction chemotherapy followed by EPP. Despite a novel finding, for a rare disease such as MPM, it is of particular importance to validate this finding in a larger patient cohort together with other clinical prognostic markers.

The authors thank Dr M. Friess for data management, analysis, and manuscript preparation; Dr Svenja Thies for scoring the TMAs for ERCC1 expression; Martina Storz for technical assistance with TMA construction; and Dr Chloé Spichiger for manuscript revision. Dr P. Vogt and the Zurich Pneumoconiosis research group at the Swiss Federal Institute of Technology (ETHZ, Director: M. Rueegger) are acknowledged for providing archival material. The authors thank the following Institutes of Pathology for providing archival paraffin blocks: University Hospital Bern (Professor Perren), University Hospital Basel (Professor Bubendorf), Cantonal Hospital St Gallen (Professor W. Jochum),
Cantonal Hospital Luzern (Professor J. Diebold), City Hospital Triemli (Professor P. Komminoth), Cantonal Hospital Aarau (Professor Grobholz), Cantonal Hospital Baden (Professor G. Singer), Cantonal Hospital Winterthur (Dr R. Flury), Cantonal Hospital Chur (Professor Stallmach), and Cantonal Hospital Münsterlingen (Dr C. Moll). The authors thank Krebsliga Zurich and the Swiss National Foundation for financial support for this study with a grant to Dr Opitz.

\section{Conflict of Interest Statement}

Rolf Stahel reports consulting fees from Astellus Pharma, Eli Lilly, Medtronic, and Roche and equity ownership in Boehringer-Ingelheim. All other authors have nothing to disclose with regard to commercial support.

You can watch a Webcast of this AATS meeting presentation by going to: http://webcast.aats.org/2014/files/ Tuesday/20140429_435PM_455PM_Isabelle_Opitz.mp4

\section{References}

1. Rusch VW, Giroux D, Kennedy C, Ruffini E, Cangir AK, Rice D, et al. Initial analysis of the international association for the study of lung cancer mesothelioma database. J Thorac Oncol. 2012;7:1631-9.

2. Rusch V, Baldini EH, Bueno R, De Perrot M, Flores R, Hasegawa S, et al. The role of surgical cytoreduction in the treatment of malignant pleural mesothelioma: meeting summary of the International Mesothelioma Interest Group Congress, September 11-14, 2012, Boston, Mass. J Thorac Cardiovasc Surg. 2013; 145:909-10.

3. Weder W, Stahel RA, Bernhard J, Bodis S, Vogt P, Ballabeni P, et al. Multicenter trial of neo-adjuvant chemotherapy followed by extrapleural pneumonectomy in malignant pleural mesothelioma. Ann Oncol. 2007;18:1196-202.

4. Weder W. Mesothelioma. Ann Oncol. 2010;21:326-33.

5. Kaufman AJ, Flores RM. Surgical treatment of malignant pleural mesothelioma. Curr Treat Options Oncol. 2011;12:201-16.

6. Besse B, Olaussen KA, Soria JC. ERCC1 and RRM1: ready for prime time? J Clin Oncol. 2013;31:1050-60.

7. Simon GR, Sharma S, Cantor A, Smith P, Bepler G. ERCC1 expression is a predictor of survival in resected patients with non-small cell lung cancer. Chest. 2005; 127:978-83.

8. Olaussen KA, Dunant A, Fouret P, Brambilla E, Andre F, Haddad V, et al. DNA repair by ERCC1 in non-small-cell lung cancer and cisplatin-based adjuvant chemotherapy. N Engl J Med. 2006;355:983-91.

9. Gautam A, Bepler G. Suppression of lung tumor formation by the regulatory subunit of ribonucleotide reductase. Cancer Res. 2006;66:6497-502.

10. Kao SC, Lee K, Klebe S, Henderson D, McCaughan B, Vardy J, et al. Excision repair cross complementation group 1 and thymidylate synthase expression in patients with mesothelioma. Clin Lung Cancer. 2013;14:164-71.

11. Zimling ZG, Sørensen JB, Gerds TA, Bech C, Andersen CB, Santoni-Rugiu E Low ERCC1 expression in malignant pleural mesotheliomas treated with cisplatin and vinorelbine predicts prolonged progression-free survival. $J$ Thorac Oncol. 2012;7:249-56.

12. Zucali PA, Giovannetti E, Destro A, Mencoboni M, Ceresoli GL, Gianoncelli L, et al. Thymidylate synthase and excision repair cross-complementing group-1 as predictors of responsiveness in mesothelioma patients treated with pemetrexed/ carboplatin. Clin Cancer Res. 2011;17:2581-90.

13. de Perrot M, Feld R, Cho BC, Bezjak A, Anraku M, Burkes R, et al. Trimodality therapy with induction chemotherapy followed by extrapleural pneumonectomy and adjuvant high-dose hemithoracic radiation for malignant pleural mesothelioma. J Clin Oncol. 2009;27:1413-8.

14. Kononen J, Bubendorf L, Kallioniemi A, Bärlund M, Schraml P, Leighton S, et al. Tissue microarrays for high-throughput molecular profiling of tumor specimens. Nat Med. 1998;4:844-7.

15. Byrne MJ, Nowak AK. Modified RECIST criteria for assessment of response in malignant pleural mesothelioma. Ann Oncol. 2004;15:257-60.

16. Bitanihirwe BK, Meerang M, Friess M, Soltermann A, Frischknecht L, Thies S, et al. PI3K/mTOR signaling in mesothelioma patients treated with induction 
chemotherapy followed by extrapleural pneumonectomy. J Thorac Oncol. 2014; 9:239-47.

17. Dong X, Hao Y, Wei Y, Yin Q, Du J, Zhao X. Response to first-line chemotherapy in patients with non-small cell lung cancer according to RRM1 expression. PLoS One. 2014;9:e92320.

18. Rosell R, Scagliotti G, Danenberg KD, Lord RV, Bepler G, Novello S, et al. Transcripts in pretreatment biopsies from a three-arm randomized trial in metastatic non-small-cell lung cancer. Oncogene. 2003;22:3548-53.

19. Rosell R, Felip E, Taron M, Majo J, Mendez P, Sanchez-Ronco M, et al. Gene expression as a predictive marker of outcome in stage IIB-IIIA-IIIB non-small cell lung cancer after induction gemcitabine-based chemotherapy followed by resectional surgery. Clin Cancer Res. 2004;10(12 Pt 2):4215s-9s.

20. Jordheim LP, Seve P, Tredan O, Dumontet C. The ribonucleotide reductase large subunit (RRM1) as a predictive factor in patients with cancer. Lancet Oncol. 2011;12:693-702.

21. Bepler G, Kusmartseva I, Sharma S, Gautam A, Cantor A, Sharma A, et al. RRM1 modulated in vitro and in vivo efficacy of gemcitabine and platinum in non-small-cell lung cancer. J Clin Oncol. 2006;24:4731-7.

22. Bepler G, Olaussen KA, Vataire AL, Soria JC, Zheng Z, Dunant A, et al. ERCC1 and RRM1 in the international adjuvant lung trial by automated quantitative in situ analysis. Am J Pathol. 2011:178:69-78.

23. Xue L, Zhou B, Liu X, Qiu W, Jin Z, Yen Y. Wild-type p53 regulates human ribonucleotide reductase by protein-protein interaction with p53R2 as well as hRRM2 subunits. Cancer Res. 2003;63:980-6.

24. Jakobsen JN, Santoni-Rugiu E, Sorensen JB. Differences in RRM1 protein expression between diagnostic biopsies and resection specimens, and changes during carboplatin and paclitaxel treatment, in non-small-cell lung cancer. Histopathology. 2014;64:412-20.

25. Yokomise H, Liu D, Ishikawa S, Go T, Gotoh M, Okuda M, et al. Chemotherapy followed by surgery on the basis of biomarker examination for patients with advanced non-small cell lung cancer. Anticancer Res. 2013;33:5597-602.

26. Bergman AM, Eijk PP, Ruiz van Haperen VW, Smid K, Veerman G, Hubeek I, et al. In vivo induction of resistance to gemcitabine results in increased expression of ribonucleotide reductase subunit M1 as the major determinant. Cancer Res. 2005;65:9510-6.

27. Zimling ZG, Sorensen JB, Gerds TA, Bech C, Andersen CB, Santoni-Rugiu E. A biomarker profile for predicting efficacy of cisplatin-vinorelbine therapy in malignant pleural mesothelioma. Cancer Chemother Pharmacol. 2012;70:743-54.

28. Ting S, Mairinger FD, Hager T, Welter S, Eberhardt WE, Wohlschlaeger J, et al. ERCC1, MLH1, MSH2, MSH6, and betallI-tubulin: resistance proteins associated with response and outcome to platinum-based chemotherapy in malignant pleural mesothelioma. Clin Lung Cancer. 2013;14:558-67.e3.

29. Friboulet L, Olaussen KA, Pignon JP, Shepherd FA, Tsao MS, Graziano S, et al. ERCC1 isoform expression and DNA repair in non-small-cell lung cancer. $N$ Engl J Med. 2013;368:1101-10.

30. Erculj N, Kovac V, Hmeljak J, Franko A, Dodic-Fikfak M, Dolzan V. The influence of gemcitabine pathway polymorphisms on treatment outcome in patients with malignant mesothelioma. Pharmacogenet Genomics. 2012;22:58-68.

Key Words: ERCC1, RRM1, mesothelioma, multimodality treatment, prognostic factor

\section{Discussion}

Dr H. Pass (New York, NY). This excellent presentation and article touch on many of the controversial elements of multimodality therapy for pleural mesothelioma. We continue to search for patient or tumor characteristics that may tell us that this patient is a reasonable one from a toxicity and efficacy standpoint for aggressive management as defined by the use of surgery and neo or postsurgical adjuvant therapy.

The authors inform us that immunohistochemical evidence of an elevated nuclear RRM1 level is associated with prolonged progression-free survival and OS in patients receiving induction chemotherapy, EPP, and postoperative radiation therapy as part of their pioneering phase II trials. This novel finding, along with validation that low levels of ERCC1 are associated with tumor response and increased survival, makes this an important contribution and certainly invites validation by the major players on the mesothelioma circuit. However, one must beware and not take this pathway and the technical aspects of the pathway too lightly. This particular study uses standard immunohistochemical methods, H-score, for the standard quantification of the gene product, protein, whereas others have used more specific quantitational methods, including AQUA, for simultaneous quantitation of ERCC1 and RRM1. Moreover, others have used polymerase chain reaction to quantify cytoplasmic and nuclear expression. Another consideration is that these particular genes have many polymorphisms, and they may have different functions depending on which polymorphism. However, prognostic factors don't work in a vacuum, and in many studies, including what you mentioned from Dr Rusch, stage, histology, and gender are all mentioned as independent prognostic factors.

My first question relates to this in that the published multimodal trials vary with regard to prognostic factors, including response to chemotherapy, presence of any metastatic nodes, and gender. Your group has presented some of these data previously and has developed the Zurich score for prognosis, which includes tumor volume, C-reactive protein level, and progression after treatment and histology. So my first question is, does nuclear RRM1, as a dichotomous value or in a continuous mode, factor as an independent predictor of performance-free survival and OS when you use these other clinical factors that I have mentioned that have been used in the literature?

Dr Opitz. Regarding your question concerning tumor volume, we did not have this variable available for the entire patient cohort because it is mainly a patient cohort from an earlier era. We had tumor volume for only a small subset of patients, so it didn't make sense to include this important variable in the multivariate model. The parameters I have been showing you were the ones that had an influence on progression-free survival in univariate analysis, and so we included only those beside the DNA markers, and this is the result. We observed no influence of RRM1 and ERCC1 on OS in the univariate analysis, so we didn't go further for a multivariate model.

Dr Pass. You have an interesting finding that you actually had a change in the cytoplasmic level of RRM1 before and after therapy. Instead of looking at the absolute value of RRM1 before and after, did you look at the change to see if that had an influence on your progression-free or OS?

Dr Opitz. We were also interested if this expression change of the marker profile has a prognostic impact, as we observed in a previous study that the change of marker profiles during chemotherapy indeed has a prognostic impact, but for the DNA marker profile change we were not able to prove a significant influence on overall or progression-free survival.

Dr Pass. Have you combined these 2 to see if you have an additive effect with both markers?

Dr Opitz. Yes, we have combined both markers and observed an additive effect, and we have to include that in the article too.

Dr Pass. Yes, you do.

Dr Opitz. If you combine both factors, we observed an even bigger difference in survival.

Dr Pass. My next question has to do with the comparison of populations. In your article, you mention a nonsurgical, older population who did not receive much therapy, and that was sort of a comparison. At least when you presented your data, you have at least 50 patients who received induction therapy but never 
underwent an EPP that you have described in the literature. Have you looked at those patients to see in the nonsurgical situation whether these markers have the same prognostic and predictive value?

Dr Opitz. Of the 50 patients who did not undergo EPP, there were only 14 who had no surgery at all. There was another subset who had pleurectomies or just an exploratory thoracotomy. Because there were only 14 patients without surgery, we did not want to perform statistics on this small subset of patients. But we will now have access to a larger collection of samples, and we can also look at another intention-to-treat population for the prognostic role of the markers for these patients.

Dr Pass. What is the next step? What is your next protocol to try and convince us all to start using this?

Dr Opitz. As you mentioned, our Multimodality Benefit Score, which is currently validated in a second cohort, is a score that uses variables that are available before the decision making for surgery, such as the tumor volume, pretreatment CRP, histologic subtype, and response to chemotherapy. We are further evaluating this score because we think it is not a single factor that should predict the outcome of the patient, and ERCC1 and RRM1 might be other variables to include in this score.
Dr A. Turna (Istanbul, Turkey). My question follows Dr Pass's first question. There are 2 factors that were shown to be prognostic factors before: the type of surgery and the fluorodeoxyglucose uptake. Did you look at these 2 variables and did you use these variables for univariate and multivariate analyses to see the possible cross-relationship between each other and the possible independent impact on survival?

Dr Opitz. Regarding the type of surgery, the patient cohort of the 107 patients analyzed all underwent EPP, no other surgical intervention. Harvey was just talking about the whole population at our institution, if we had a look at these other patients. Regarding fluorodeoxyglucose uptake, we were not prospectively including fluorodeoxyglucose uptake for this patient cohort in our database, so no, but we are including this variable now in our documentation.

Dr M. Krasna (Neptune, $N J$ ). Of the 32 patients who received pemetrexed, did they receive pemetrexed together with platinum or was it in lieu of platinum, and in those patients did you check for other markers for sensitivity or resistance to pemetrexed, such as thymidylate synthase or others?

Dr Opitz. No, we did not assess other markers for sensitivity or resistance.

Readers who found these articles interesting may also like to read the following papers found in recent and future issues of our sister publications, Seminars in Thoracic and Cardiovascular Surgery and Operative Techniques in Thoracic and Cardiovascular Surgery!

\section{General Thoracic Surgery: Other Thoracic Surgical Papers}

Current Readings: David Zeltsman. Redefining Minimally Invasive Uniportal Video-Assisted Thoracic Surgery. Semin Thorac Cardiovasc Surg. Autumn 2014;26(3):249-254.

Current Readings: Sumeet Mittal. Failed Hiatus Hernia Repair. Semin Thorac Cardiovasc Surg. 2014;26(4):331-334.

State of the Art: Frank Detterbeck. The ITMIG Thymic Initiative: A State of the Art for the Study of Thymic Malignancies. Semin Thorac Cardiovasc Surg. 2014;26(4):317-322.

State of the Art: Richard Freeman. The Theory, Practice and Future of Process Improvement in General Thoracic Surgery. Semin Thorac Cardiovasc Surg. 2014;26(4):310-316.

State of the Art: Eric Grogan. Using Clinical Risk Models for Lung Nodule Classification. Semin Thorac Cardiovasc Surg. Expected Publication August 2015.

Original Submission: Thomas Watson. Eliminating a need for esophagectomy: endoscopic treatment of Barrett's Esophagus with early esophageal neoplasia. Semin Thorac Cardiovasc Surg. 2014;26(4):274-284.

Editorial Commentary: Anthony Kim. Endoscopically closing doors. Semin Thorac Cardiovasc Surg. 2014;26(4):285-286.

Original Submission: Daniela Molena. Incidence and risk factors for respiratory complications in patients undergoing esophagectomy for malignancy: a NSQIP analysis. Semin Thorac Cardiovasc Surg. 2014;26(4):287-294.

Editorial Commentary: R. Taylor Ripley. Esophageal complications: What are the real results? Semin Thorac Cardiovasc Surg. Expected publication April 2015. 2014;26(4):295-296.

Otis Rickman. Electromagnetic Navigation Assisted Bronchoscopy. Oper Tech Thorac Cardiovasc Surg. 2014;19(2):199-218.

Eric Grogan. Localization techniques for small lung nodules. Oper Tech Thorac Cardiovasc Surg. 2014;19(2):179-198.

Donald Nuss. The Minimally Invasive Repair of Pectus Excavatum. Oper Tech Thorac Cardiovasc Surg. 2014;19(3):324-347.

John Kucharczuk. Total Pectus Excavatum Repair: Open Approach. Oper Tech Thorac Cardiovasc Surg. 2014;19(3):348-364. 


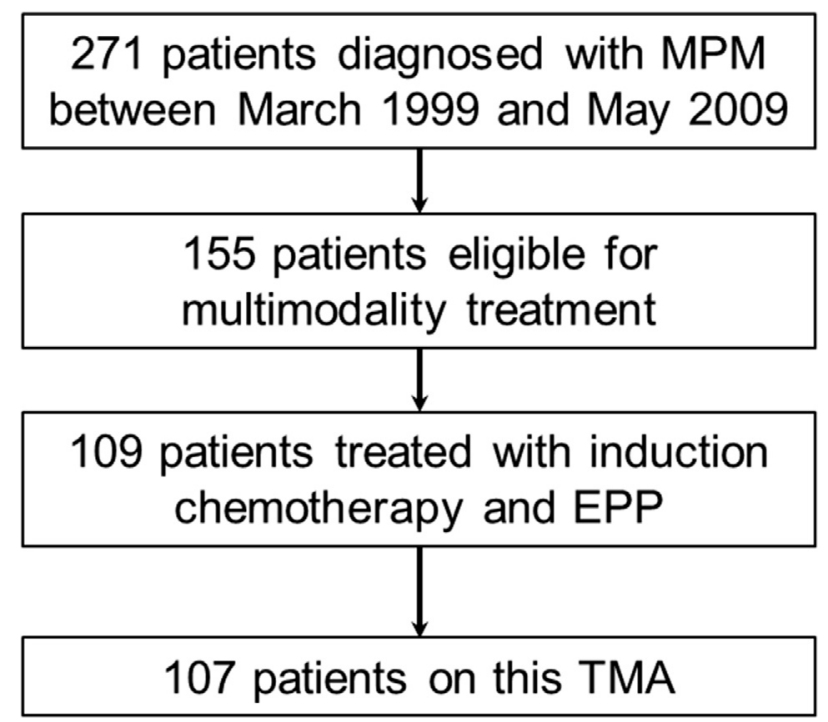

FIGURE E1. Consort graph of the patient cohort. EPP, Extrapleural pneumonectomy; MPM, malignant pleural mesothelioma; TMA, tissue microarray.

TABLE E1. Expression of ribonucleotide reductase M1 and excision repair cross-complementation group 1 in pre- and postchemotherapy specimens

\begin{tabular}{lccccccc}
\hline & \multicolumn{3}{c}{ Prechemotherapy } & & \multicolumn{3}{c}{ Postchemotherapy } \\
\cline { 2 - 4 } H-score & Cytoplasmic RRM1 & Nuclear RRM1 & Nuclear ERCC1 & & Cytoplasmic RRM1 & Nuclear RRM1 & Nuclear ERCC1 \\
\hline 0.00 & $0(0 \%)$ & $36(51 \%)$ & $10(12 \%)$ & & $2(2 \%)$ & $83(84 \%)$ & $0(0 \%)$ \\
$0.01-1.00$ & $59(83 \%)$ & $14(20 \%)$ & $7(8 \%)$ & & $44(44 \%)$ & $10(10 \%)$ & $19(18 \%)$ \\
$1.01-2.00$ & $10(14 \%)$ & $13(18 \%)$ & $33(38 \%)$ & & $44(44 \%)$ & $5(5 \%)$ & $56(54 \%)$ \\
$2.01-3.00$ & $2(3 \%)$ & $8(11 \%)$ & $37(42 \%)$ & & $9(9 \%)$ & $1(1 \%)$ & $29(28 \%)$ \\
\hline
\end{tabular}

Frequency distribution of biomarker expression: no $(\mathrm{H}$-score $=0.00)$, low $(\mathrm{H}$ score $=0.01-1.00)$, moderate $(\mathrm{H}$ score $=1.01-2.00)$, and strong $(\mathrm{H}$ score $=2.01-3.00)$. Data are given in numbers and percent. RRM1, Ribonucleotide reductase M1; ERCC1, excision repair cross-complementation group 1. 